\title{
Article \\ Cerebellar White Matter Abnormalities in Charcot-Marie-Tooth Disease: A Combined Volumetry and Diffusion Tensor Imaging Analysis
}

\author{
Sungeun Hwang ${ }^{1,+}+\mathbb{D}$, Chang-Hyun Park ${ }^{2,+}$, Regina Eun-Young Kim ${ }^{3,4,+}$, Hyeon Jin Kim ${ }^{2,5,+}+\mathcal{D}$, Yun Seo Choi ${ }^{6}$, \\ Sol-Ah Kim ${ }^{6}$, Jeong Hyun Yoo ${ }^{7}$, Ki Wha Chung ${ }^{8}$ (D) Byung-Ok Choi ${ }^{9,10,11, * \mathbb{C}}$ and Hyang Woon Lee ${ }^{1,2,6,12,13, * \mathbb{D}}$
}

\section{check for}

updates

Citation: Hwang, S.; Park, C.-H.; Kim, R.E.-Y.; Kim, H.J.; Choi, Y.S.; Kim, S.-A.; Yoo, J.H.; Chung, K.W.; Choi, B.-O.; Lee, H.W. Cerebellar White Matter Abnormalities in Charcot-Marie-Tooth Disease: A Combined Volumetry and Diffusion Tensor Imaging Analysis. J. Clin. Med. 2021, 10, 4945. https://doi.org/ $10.3390 / \mathrm{jcm} 10214945$

Academic Editor: Daniele Orsucci

Received: 11 September 2021

Accepted: 21 October 2021

Published: 26 October 2021

Publisher's Note: MDPI stays neutral with regard to jurisdictional claims in published maps and institutional affiliations.

Copyright: (C) 2021 by the authors. Licensee MDPI, Basel, Switzerland. This article is an open access article distributed under the terms and conditions of the Creative Commons Attribution (CC BY) license (https:// creativecommons.org/licenses/by/ $4.0 /)$.
1 Departments of Neurology, Ewha Womans University Mokdong Hospital, Seoul 07985, Korea; neurosung@gmail.com

2 Departments of Neurology, Ewha Womans University School of Medicine and Ewha Medical Research Institute, Seoul 07985, Korea; park.changhyun@hanmail.net (C.-H.P.); hkimneurology@gmail.com (H.J.K.)

3 Institute for Human Genomic Study, College of Medicine, Korea University, Ansan 15355, Korea; eunyoung.regina.kim@gmail.com

4 Department of Psychiatry, University of Iowa, Iowa City, IA 52242, USA

5 Department of Neurology, Korea University Ansan Hospital, Ansan 15355, Korea

6 Departments of Medical Science, Ewha Womans University School of Medicine and Ewha Medical Research Institute, Seoul 07804, Korea; coolgirldew@naver.com (Y.S.C.); solak1221@naver.com (S.-A.K.)

7 Department of Radiology, College of Medicine, Ewha Womans University, Seoul 07985, Korea; yoolee@ewha.ac.kr

8 Department of Biological Sciences, Kongju National University, Kongju 32588, Korea; kwchung@kongju.ac.kr

9 Department of Neurology, Samsung Medical Center, Sungkyunkwan University School of Medicine, Seoul 06351, Korea

10 Department of Health Sciences and Technology, Samsung Advanced Institute for Health Sciences \& Technology, Sungkyunkwan University, Seoul 06351, Korea

11 Stem Cell \& Regenerative Medicine Institute, Samsung Medical Center, Seoul 06351, Korea

12 Department of Computational Medicine, Ewha Womans University, Seoul 07985, Korea

13 Department of System Health Science \& Engineering, Ewha Womans University, Seoul 03765, Korea

* Correspondence: bochoi@skku.edu (B.-O.C.); leeh@ewha.ac.kr (H.W.L.); Tel.: +82-2-3410-1296 (B.-O.C.); +82-2-2650-2673 (H.W.L.); Fax: +82-2-3410-0052 (B.-O.C.); +82-2-2650-5958 (H.W.L.)

+ These authors contributed equally to this work as co-first authors.

Abstract: Charcot-Marie-Tooth disease (CMT) is a genetically heterogeneous hereditary peripheral neuropathy. Brain volumetry and diffusion tensor imaging (DTI) were performed in 47 controls and 47 CMT patients with PMP22 duplication $(n=10)$, MFN2 $(n=15)$, GJB1 $(n=11)$, or NEFL mutations $(n=11)$ to investigate for structural changes in the cerebellum. Volume of cerebellar white matter (WM) was significantly reduced in CMT patients with NEFL mutations. Abnormal DTI findings were observed in the superior, middle, and inferior cerebellar peduncles, predominantly in NEFL mutations and partly in GJB1 mutations. Cerebellar ataxia was more prevalent in the NEFL mutation group (72.7\%) than the GJB1 mutation group (9.1\%) but was not observed in other genotypic subtypes, which indicates that structural cerebellar abnormalities were associated with the presence of cerebellar ataxia. However, NEFL and GJB1 mutations did not affect cerebellar gray matter (GM), and neither cerebellar GM nor WM abnormalities were observed in the PMP22 duplication or MFN2 mutation groups. We found structural evidence of cerebellar WM abnormalities in CMT patients with NEFL and GJB1 mutations and an association between cerebellar WM involvement and cerebellar ataxia in these genetic subtypes, especially in the NEFL subgroup. Therefore, we suggest that neuroimaging, such as MRI volumetry or DTI, for CMT patients could play an important role in detecting abnormalities of cerebellar WM.

Keywords: Charcot-Marie-Tooth disease (CMT); volumetry; diffusion tensor imaging (DTI); white matter; cerebellum; ataxia 


\section{Introduction}

Charcot-Marie-Tooth disease (CMT) is a hereditary peripheral neuropathy with clinical and genetic heterogeneities [1]. Peripheral myelin protein 22 (PMP22) duplication is known to cause CMT1A, while mitofusin 2 (MFN2) mutations cause CMT2A and gap junction protein 1 (GJB1) mutations are relevant to CMTX1 [2]. Mutations in the neurofilament light chain polypeptide (NEFL) produce a variety of CMT phenotypic spectra, including CMT1F, CMT2E, dominant intermediate CMT G (CMTDIG), and autosomal recessive CMT [3].

However, the central nervous system (CNS) has been implicated in several CMT patients with GJB1 and MFN2 mutations [4,5], and CNS symptoms have been reported in CMT patients with NEFL mutations [6,7]. Cerebellar ataxia is one of the common symptoms of cerebellar dysfunction, which manifests as an inability to control or coordinate voluntary movements, with disturbance in planning and execution of movements [8]. Cerebellar ataxia has been reported in some NEFL patients [6,7,9-11]. From the literature review, among 173 patients with NEFL mutations, ataxia was found in 22 patients and cerebellar atrophy in 4 patients [6]. Even in patients who have been reported to have cerebellar ataxia, it is difficult to detect cerebellar atrophy using routine conventional brain MRI. Recently, diffusion tensor imaging (DTI) study has been widely used in the neuroscience field. DTI is employed to examine white matter microstructure, such as diffusion constraints that exist within the voxel; that is, at a spatial scale well below that at which the image acquisition resolution can be identified. Whereas the sensitivity of a conventional structural MRI depends on the spatial resolution used for imaging, the sensitivity of DTI relies on its diffusion measurement of water molecules on a microscopic scale (up to $\mu \mathrm{m}$ ), irrespective of the spatial resolution used for imaging (up to $\mathrm{mm}$ ). In hereditary ataxias, DTI study has shown to be useful, revealing white matter abnormalities, and this change is associated with the severity of ataxia [12-15]. We previously described the DTI abnormality of cerebral white matter (WM) that correlated with clinical disability in CMT patients with MFN2, GJB1, and NEFL mutations [10]. Additionally, there has been a report of a combined structural and diffusion MRI study of CMT1A patients [16]. However, there has been no report of volumetry or DTI studies of the cerebellum in patients with NEFL mutations. We employed DTI to assess changes in white matter microstructure by leveraging its sensitivity to diffusion measurement on a microscopic scale.

In this study, we used both volumetry and DTI to search for structural changes in the cerebella of 47 controls and 47 CMT patients with PMP22, MFN2, GJB1, and NEFL mutations. Interestingly, we observed significant volumetric changes in the cerebella of CMT patients, especially with NEFL mutations, which were associated with the presence of cerebellar ataxia in this genetic subgroup.

\section{Materials and Methods}

\subsection{Participants}

We enrolled 94 study participants, including 47 healthy controls and 47 CMT patients: 10 CMT1A patients with PMP22 duplication, 15 CMT2A patients with MFN2 mutations, 11 CMTX1 patients with GJB1 mutations, and 11 CMT patients (3 CMT1F, 2 CMT2E, and 6 CMTDIG) with NEFL mutations (Table 1). Except one L312P NEFL patient with cerebellar atrophy, the cerebella showed normal findings in a routine conventional brain MRI. The determination of causative mutations and clinical assessments were performed as described previously [10].

\subsection{Clinical Assessments}

Clinical assessments were obtained by detailed history-taking and physical examination to assess muscle weakness, atrophy, sensory loss, and deep tendon reflexes. In order to determine physical disability, we used three scales: the Medical Research Council (MRC) scale for muscle strength [17], the functional disability scale (FDS) [18], and the CMT neuropathy score (CMTNS) [19]. Flexor and extensor muscle strength was evaluated 
using the standard MRC scale. Age at onset was determined by asking patients at what age their symptoms first appeared. Ataxia was scored by an experienced neurologist according to the scale for the assessment and rating of ataxia (SARA) while subjects performed each task. SARA is a clinical scale developed by Schmitz-Hübsch and colleagues [20] that assesses a range of different impairments in ataxia, ranging from speech to balance. The scale comprises 8 categories: gait ( $0-8$ points), stance ( $0-6$ points), sitting ( $0-4$ points), speech disturbance ( $0-6$ points), finger chase ( $0-4$ points), nose-finger test ( $0-4$ points), fast alternating hand movement ( $0-4$ points), and heel-shin slide ( $0-4$ points). Once the clinician assesses each of the 8 categories for an individual, they can further compute the cumulative score, ranging from 0 (no ataxia) to 40 (most severe ataxia), to determine the ataxic subject's severity of ataxia.

Table 1. Basic demographic and clinical characteristics of study population.

\begin{tabular}{|c|c|c|c|c|c|}
\hline & Normal Control & $\begin{array}{c}\text { PMP22 } \\
\text { Duplication }\end{array}$ & $\begin{array}{c}\text { MFN2 } \\
\text { Mutations }\end{array}$ & $\begin{array}{c}G J B 1 \\
\text { Mutations }\end{array}$ & $\begin{array}{c}\text { NEFL } \\
\text { Mutations }\end{array}$ \\
\hline Number & 47 & 10 & 15 & 11 & 11 \\
\hline Female (\%) & 49 & 45 & 60 & 45 & 36 \\
\hline $\begin{array}{l}\text { Age at exam } \\
\text { (years) }\end{array}$ & $37.5 \pm 14.6$ & $42.0 \pm 14.7$ & $31.9 \pm 13.6$ & $39.4 \pm 16.5$ & $39.5 \pm 11.8$ \\
\hline $\begin{array}{l}\text { Age at onset } \\
\text { (years) }\end{array}$ & - & $11.2 \pm 6.9$ & $10.6 \pm 11.3$ & $23.5 \pm 13.7$ & $14.0 \pm 4.5$ \\
\hline Muscle weakness & No & $\mathrm{UL}<\mathrm{LL}^{\mathrm{a}}$ & $\mathrm{UL}<\mathrm{LL}$ & $\mathrm{UL}<\mathrm{LL}$ & $\mathrm{UL}<\mathrm{LL}$ \\
\hline Sensory loss & No & Yes & Yes & Yes & Yes \\
\hline $\operatorname{MRC}^{\mathrm{b}}(\mathrm{arm})$ & 5 & $3.9 \pm 0.3$ & $1.6 \pm 1.7$ & $3.8 \pm 0.9$ & $2.1 \pm 0.7$ \\
\hline MRC $^{c}$ (leg) & 5 & $2.1 \pm 1.2$ & $1.4 \pm 1.3$ & $3.5 \pm 1.3$ & $1.8 \pm 0.6$ \\
\hline FDS $^{d}$ & 0 & $2.0 \pm 1.1$ & $3.9 \pm 2.2$ & $2.3 \pm 1.0$ & $3.0 \pm 1.0$ \\
\hline CMTNS v2 ${ }^{e}$ & 0 & $14.3 \pm 5.5$ & $18.7 \pm 8.9$ & $12.6 \pm 6.3$ & $14.7 \pm 5.7$ \\
\hline Cerebellar ataxia & 0 & 0 & 0 & $1(9.1 \%)$ & $8(72.7 \%)$ \\
\hline SARA ${ }^{\mathrm{f}}$ & 0 & 0 & 0 & 7.0 & $10.6 \pm 3.4$ \\
\hline \multicolumn{6}{|c|}{ Peripheral ulnar nerve conduction studies } \\
\hline CMAPg $(\mathrm{mV})$ & $15.9 \pm 2.8$ & $6.6 \pm 3.5$ & $7.2 \pm 6.0$ & $9.3 \pm 3.0$ & $5.8 \pm 4.8$ \\
\hline $\operatorname{MNCV}^{\mathrm{h}}(\mathrm{m} / \mathrm{s})$ & $61.2 \pm 3.2$ & $19.6 \pm 3.9$ & $52.3 \pm 8.4$ & $43.3 \pm 9.0$ & $36.0 \pm 9.0$ \\
\hline $\mathrm{SNAP}^{\mathrm{i}}(\mathrm{uV})$ & $23.1 \pm 7.2$ & $5.2 \pm 1.7$ & $8.4 \pm 4.8$ & $5.8 \pm 3.3$ & $4.3 \pm 2.1$ \\
\hline $\mathrm{SNCV}^{\mathrm{j}}(\mathrm{m} / \mathrm{s})$ & $51.6 \pm 2.5$ & $21.3 \pm 4.8$ & $32.9 \pm 5.8$ & $30.5 \pm 3.2$ & $36.3 \pm 3.8$ \\
\hline
\end{tabular}

${ }^{a}$ UL < LL, upper limb weakness < lower limb weakness; ${ }^{b}$ MRC (arm), Medical Research Council scale for motor weakness from finger abduction; ${ }^{\mathrm{c}}$ MRC (leg), Medical Research Council scale for motor weakness from ankle dorsiflexion; ${ }^{\mathrm{d}}$ FDS, functional disability scale; e CMTNS v2, Charcot-Marie-Tooth neuropathy score version 2; ${ }^{\mathrm{f}}$ SARA scale is made up of 8 categories with scores ranging as, gait (0-8 points), stance ( $0-6$ points), sitting ( $0-4$ points), speech disturbance ( $0-6$ points), finger chase ( $0-4$ points), nose-finger test (0-4 points), fast alternating hand movement (0-4 points), heel-shin slide ( $0-4$ points); ${ }^{\mathrm{C}} \mathrm{CMAP}$, compound muscle action potential; ${ }^{\mathrm{h}} \mathrm{MNCV}$, motor nerve conduction velocity; ${ }^{i}$ SNAP, sensory nerve action potential; ${ }^{j}$ SNCV, sensory nerve conduction velocity. Normal NCV values: ulnar motor nerve, $\geq 51.1 \mathrm{~m} / \mathrm{s}$; ulnar sensory nerve, $\geq 37.5 \mathrm{~m} / \mathrm{s}$. Normal amplitude values: ulnar motor nerve, $\geq 8 \mathrm{mV}$; ulnar sensory nerve, $\geq 7.9 \mathrm{uV}$.

\subsection{MR Volumetry and Diffusion Tensor Imaging (DTI)}

We performed 3-T MRI for volumetry and DTI with high resolution 3-dimensional T1-weighted images and diffusion-weighted EPI sequences (Philips Achieva v2.6, Best, The Netherlands). MRI processing consisted of AC-PC co-registration between T1-images, multimodal bias-correction, and applying a multi-label joint fusion algorithm. Using the BRAINSTools suite, the brain was divided into 217 sub-regions, which were then classified as either cerebral or cerebellar for further analysis. Finally, age and total intracranial volumes were adjusted to compare the WM and gray matter (GM) volumes. All volumetric data were processed through a fully automated procedure, BRAINS Auto-Workup (BAW) [21-23], improved with SyN registration from the Advanced Normalization Toolkit in the BRAINSTools suite (https:/ / github.com/BRAINSia/BRAINSTools (accessed on 31 March 2021)) [23]. The resulting data set of bias-corrected average T1 images was subsequently segmented for subcortical structures using an automated segmentation framework, 
ANTs MALF [23,24]. Both the raw data and the resultant segmentation were rigorously reviewed for validity by visual inspection.

DTI data were collected using the same parameters (TR, $4500 \mathrm{~ms}$; TE, $68 \mathrm{~ms}$; EoV RL, $240 \mathrm{~mm} \times$ AP $240 \mathrm{~mm}$; FH, $135 \mathrm{~mm}$; matrix size, $128 \times 128 \mathrm{~mm}, 3 \mathrm{~mm}$ slice thickness, no gap; flip angle, 90 degrees; voxel size: RL, 2; AP, 2; 32 directions with b-value $1000 \mathrm{~s} / \mathrm{mm}^{2}$, and one null image with $b$-value $0 \mathrm{sec} / \mathrm{mm}^{2}$; total diffusion gradient, $80 \mathrm{mT} / \mathrm{m} ; \mathrm{NSA}=1$; 45 slices in transverse plane) and analyzed using the tools included in the FSL (http:// fsl.fmrib.ox.ac.uk/fsl/ (accessed on 31 March 2021)) to compute DTI-derived measures, including fractional anisotropy (FA), axial diffusivity (AD), and radial diffusivity (RD), as described elsewhere [10]. The protocol for this study was approved by the Institutional Review Board, and informed consent was obtained from all patients and from parents of patients younger than 18 years of age.

\subsection{Statistical Analysis}

We analyzed volumetric data using $\mathrm{R}$ software version 3.3. Whole cerebellar tissue and regional cerebellar volumes were standardized to the individual intracranial volume (ICV) to account for differences in head size. We performed all analysis of volume differences using \% scale, volume per ICV in percentage [23]. Left- and right-side volumes of the cerebellum were combined. A comparison of cerebellar volumes between normal control and CMT patients for genetic subtypes was performed using Analysis of Variance (ANOVA), using age and sex as covariates. A Bonferroni correction was applied to adjust for multiple comparisons.

The tract-based spatial statistics (TBSS) approach was used for voxelwise statistical analysis, with correction for age and sex $[10,25,26]$. We compared the DTI-derived measures between control and CMT groups with genetic subtypes. The familywise error rate was controlled using the threshold-free cluster enhancement (TFCE) approach [26], by which cluster-like structures are enhanced without having to define clusters in a binary way. Group differences in DTI from TBSS were evaluated with TFCE-corrected $p<0.05$ to adjust for multiple comparisons. The FA and diffusivity measures of DTI were tested for correlation with clinical variables by using Spearman's rank correlation coefficients ( $\rho)[10,25]$.

\section{Results}

The clinical features and nerve conduction studies of the 47 CMT patients are shown in Table 1. CMT patients had muscle weakness and atrophy, predominantly in the distal legs, ranging from mild weakness to complete paralysis. Genetic mutations included eight MFN2 mutations, seven GJB1 mutations, and six NEFL mutations. CMT patients with NEFL and GJB1 mutations exhibited diverse cerebellar involvements, including cerebellar ataxia, dysarthria, dyssynergia, and dysmetria. In particular, the frequency of the cerebellar ataxia was high in patients with NEFL mutations (72.7\%), compared to GJB1 mutations (9.1\%), PMP22 duplication ( $0 \%$ ), and MFN2 mutations ( $0 \%$ ).

The mean volume proportion of the cerebellar region was $3.08 \%( \pm 0.25, \mathrm{~V} / \mathrm{ICV})$ for normal controls, while most of the CMT groups showed lower cerebellar regional volumes (Figure 1). A group comparison showed a significant difference in cerebellar GM and WM volumes in the NEFL mutation group (Figure 1A,B). The difference in cerebellar WM in the NEFL subgroup, compared with the control group, remained significant, even after multiple comparison adjustments $(-0.151 \pm 0.031, p=0.0083$, Figure 1B). The cerebellar WM volume of patients in the MFN2 group revealed a nonsignificant tendency to be reduced $(-0.051 \pm 0.028, p=0.07)$. However, there was no statistically significant cerebellar atrophy in the other genotypes (PMP22 duplication, MFN2, or GJB1 mutation). Among the NEFL subgroup, SARA showed a negative correlation with the volume proportion of cerebellar WM (Spearman's $\rho=-0.604, p=0.049$ ) but not with cerebellar GM (Spearman's $\rho=-0.346, p=0.298)$. 
(A) Volumetry of cerebellar GM

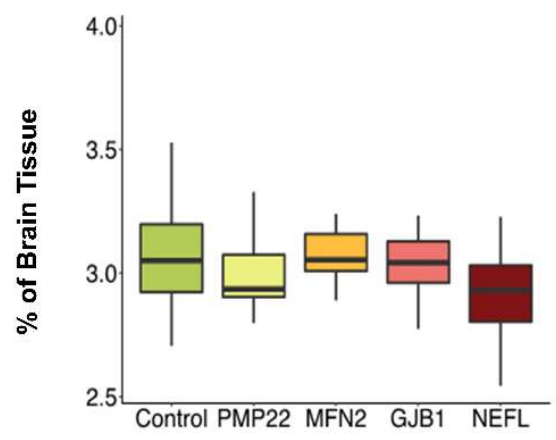

(C) Correlation between ataxia and cerebellar GM

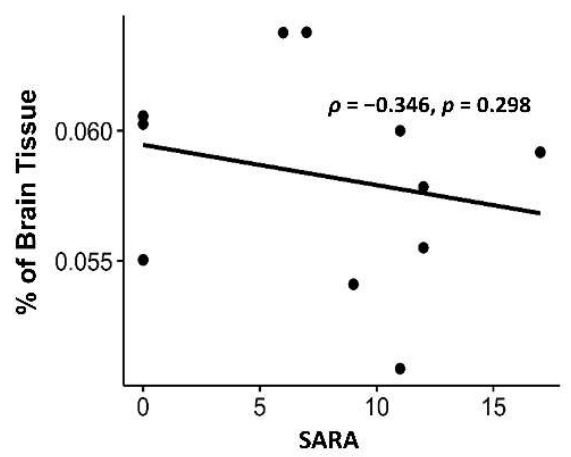

(B) Volumetry of cerebellar WM

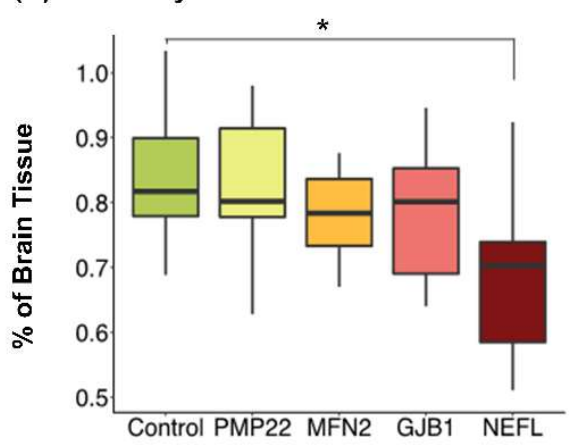

(D) Correlation between ataxia and cerebellar WM

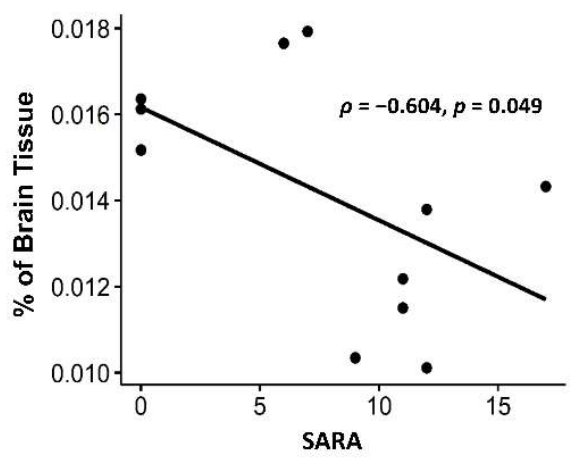

Figure 1. Volumetric findings of gray matter (A) and white matter $(\mathbf{B})$ in cerebellum of CMT patients with different genetic subtypes. CMT patients with NEFL genetic mutations demonstrate distinct decreased volumetric changes in cerebellar white matter (B) but not cerebellar gray matter (A), while neither cerebellar GM nor WM changes were observed in the subgroups with PMP22 duplication, MFN2, or GJB1 mutations. (C) Correlation between SARA and cerebellar GM in NEFL subgroup. (D) Correlation between SARA and cerebellar WM in NEFL subgroup. Significant difference in paired test was marked with *, $p<0.05$. Abbreviations: GM, gray matter; WM, white matter.

DTI of the cerebellum revealed significantly reduced FA and AD and increased RD values, most prominently in the NEFL genetic subgroup and mildly in GJB1 subtype, but no changes in the other subgroups with PMP22 duplication or MFN2 mutation (Figure 2). In the NEFL genetic subtype, DTI of the cerebellum revealed significantly reduced FA values in $90.9 \%$ of the voxels of the superior cerebellar peduncle (SCP), $63.4 \%$ of the middle cerebellar peduncle (MCP) voxels, and $92.0 \%$ of the inferior cerebellar peduncle (ICP) voxels (Figure 3A). Similarly, significantly deceased AD values were found in $68.0 \%, 57.0 \%$, and $57.4 \%$ of the voxels, and increased $\mathrm{RD}$ values in $79.7 \%, 40.1 \%$, and $90.9 \%$ of the voxels in the SCP, the $\mathrm{MCP}$, and the ICP, respectively (Figure 3B). In the case of GJB1 mutation, reduced FA values were found in $24.3 \%, 14.9 \%$, and $0.4 \%$ of the voxels, and increased $\mathrm{RD}$ values in $41.4 \%$, $18.3 \%$, and $1.9 \%$ of the voxels of the $\mathrm{SCP}$, the $\mathrm{MCP}$, and the ICP, respectively. $\mathrm{AD}$ values were not different from those of healthy controls. Interestingly, manifestation of cerebellar ataxia was largely restricted to the NEFL subgroup; it was observed clinically in 8 out of 11 CMT patients in the NEFL subgroup $(72.7 \%)$ but in only 1 out of 11 CMT patients $(9.1 \%)$ in the GJB1 subgroup (Figure 3A,B). Among the NEFL subgroup, SARA was correlated to voxels with decreased AD values of the SCP (Spearman's $\rho=0.926, p<0.01$ ), the MCP (Spearman's $\rho=0.848, p<0.01$ ), and the ICP (Spearman's $\rho=0.764, p<0.01$ ). Similarly, SARA was correlated to voxels with increased RD values of the SCP (Spearman's $\rho=0.678, p=0.02$ ) and the MCP (Spearman's $\rho=0.840, p<0.01$ ). Thus, abnormal DTI findings in cerebellar WM tracts, combined with reduced cerebellar WM volume, occurred predominantly in the NEFL subgroup, the subtype associated with cerebellar ataxia symptoms. 

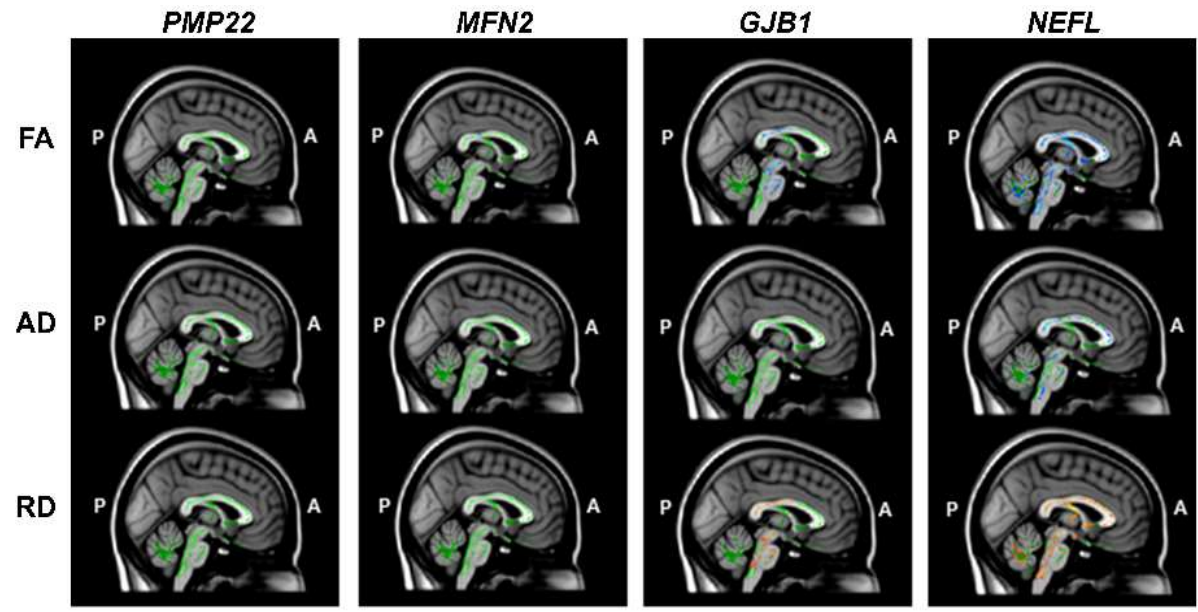

Figure 2. DTI findings in CMT patients with different genetic subtypes. DTI abnormalities, including reduced FA and $\mathrm{AD}$ and increased RD values, were observed mostly in the genetic subgroup with NEFL mutations. Decreased values depicted in blue, and increased values in red, with corrected $p<0.05$.
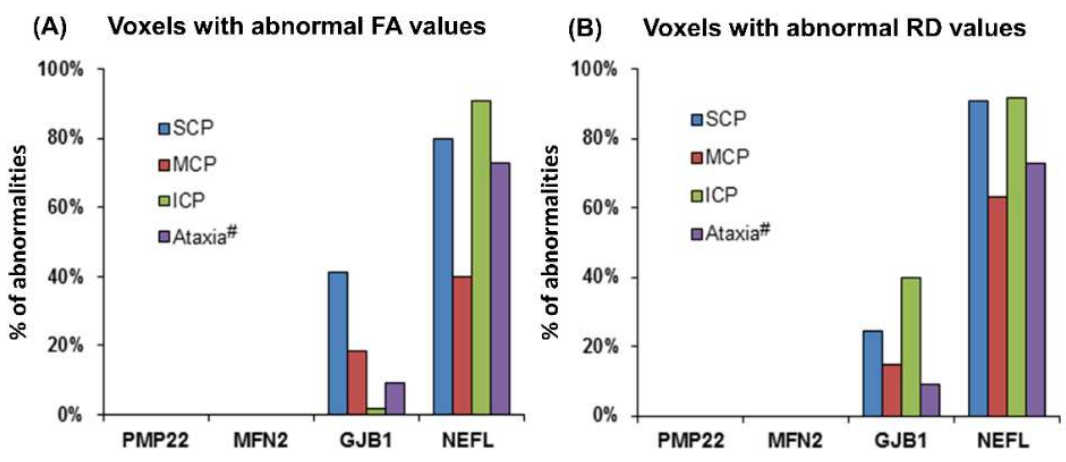

(C) Correlation between ataxia and FA

(D) Correlation between ataxia and RD
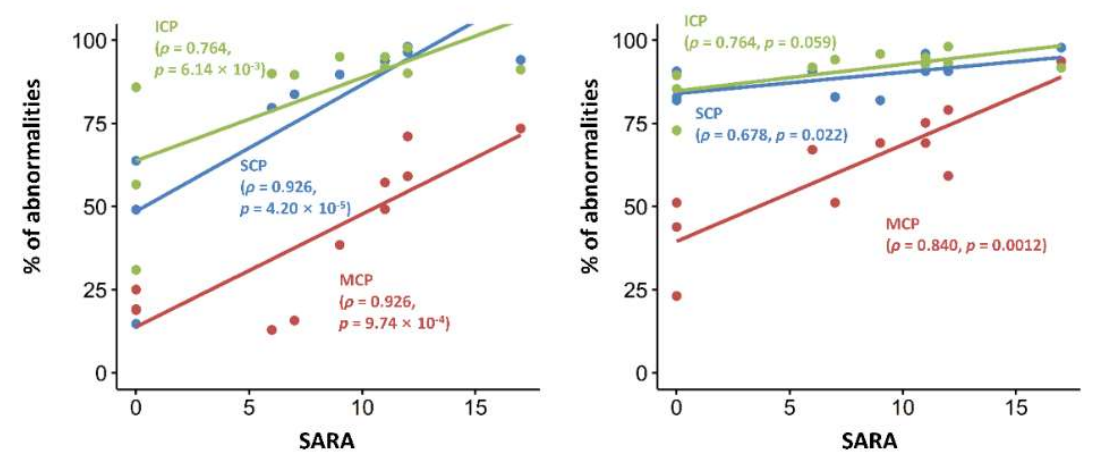

Figure 3. DTI abnormalities of FA and RD in different genetic subtypes of CMT with ataxia as a neurologic manifestation. Reduced FA (A) and increased RD (B) in major cerebellar WM tracts, including the superior cerebellar peduncle (SCP), the middle cerebellar peduncle (MCP), and the inferior cerebellar peduncle (ICP), were observed, predominantly in the NEFL subgroup, but also subtle abnormalities in the GJB1 subgroup. Interestingly, higher scores on SARA correlated with more severe abnormalities in FA (C) and RD values (D) in DTI of patients with NEFL mutation.

\section{Discussion}

In this study, we found structural evidence of cerebellar WM abnormalities, including reduced cerebellar WM by volumetry in the NEFL mutation group and microstructural abnormalities by DTI of the SCP, MCP, and ICP in the NEFL and GJB1 subgroups. It has been reported that brain T1-weighted MR images in CMT patients with the N98S NEFL mutation 
showed cerebellar atrophy [26]. It was not clear, however, whether cerebellar atrophy could be a common feature seen in the NEFL mutation in the previous studies. We examined the MRI volumetry and DTI studies in CMT patients, including NEFL mutations harboring various mutation sites. As a result, we confirmed that cerebellar atrophy is a common phenomenon observed in the NEFL mutation group. In our study, we proved that NEFL mutations affect the WM of the cerebellum but not the GM. In addition, cerebellar ataxia, which is commonly manifested in patients with NEFL mutations, is not well observed in the other genetic mutations, such as PMP22 and MFN2, except in 1 out of 11 patients with GJB1 mutations. To our knowledge, this is the first report of volumetric and DTI study of CMT patients with NEFL mutations showing cerebellar WM abnormality.

According to the literature review, in 173 patients with NEFL mutations, ataxia was found in 22 patients and cerebellar atrophy in 4 patients [6,7]. This means that some NEFL patients showed only cerebellar ataxia without cerebellar atrophy. In the present study, cerebellar ataxia was found in $72.7 \%$ of the NEFL subgroup, but cerebellar atrophy was observed in only one patient with the L312P NEFL mutation. This discrepancy may be due to the fact that ataxia caused by cerebellar dysfunction is an earlier symptom than cerebellar atrophy. Therefore, it is difficult to find cerebellar atrophy with a routine conventional brain MRI, and cerebellar involvements in NEFL patients may be underestimated. Interestingly, reduced FA and increased RD were detected in a high proportion of voxels in the SCP, MCP, and ICP in the NEFL mutation group. Furthermore, these DTI findings showed correlation with severity of cerebellar ataxia in this group. The MCP contains pontocerebellar fibers, which receive signals from corticopontine fibers, and the cerebellum, in turn, projects to the ventrolateral nucleus of the thalamus, which projects to the motor cortex to form a circuit. The ICP contains the posterior spinocerebellar tract, which relays unconscious proprioception, as well as the vestibulocerebellar tract. Thus, ataxia in the NEFL subgroup could be explained by the involvement of the SCP, MCP, and ICP.

Additionally, we found DTI cerebellar abnormalities in the GJB1 mutation group. One patient with the C179X GJB1 gene mutation showed cerebellar ataxia without cerebellar atrophy in routine conventional brain MRI. DTI of the cerebellum showed moderately reduced FA values in $24.3 \%$ of the voxels of the SCP in GJB1 group. However, it was not easy to tell whether the patient with ataxia had distinctive DTI features because there was only one patient who had relatively mild ataxia (SARA 7).

Nevertheless, MRI volumetry or DTI can be helpful for early detection of cerebellar dysfunction in CMT patients with NEFL and/or GJB1 mutations, especially in cases harboring cerebellar ataxia. In fact, GJB1 has been reported to relate to mitochondrial function in motor neurons in CNS [27] and to be expressed in both Schwann cells and oligodendrocytes, the myelinating glia of the PNS and CNS, respectively [28].

Our findings provide structural evidence for cerebellar WM involvements in CMT patients with NEFL mutations investigated by MRI volumetry and DTI studies, which is related to the prevalent manifestation of cerebellar ataxia in this genetic subtype. This study also demonstrates that quantitative MRI, such as volumetry and DTI, can be useful for clinical characterization, including future development of CNS involvement in CMT patients with diverse genetic abnormalities.

Author Contributions: Conceptualization: B.-O.C. and H.W.L.; methodology: C.-H.P., R.E.-Y.K., S.H., Y.S.C. and S.-A.K.; formal analysis and investigation: C.-H.P., R.E.-Y.K.; writing-original draft preparation: S.H., C.-H.P., R.E.-Y.K.; writing—review and editing: H.J.K., Y.S.C., S.-A.K., B.-O.C. and H.W.L.; funding acquisition: B.-O.C. and H.W.L.; resources: B.-O.C. and H.W.L.; supervision: J.H.Y. and K.W.C. All authors have read and agreed to the published version of the manuscript.

Funding: This work was supported by grants of the Korea Health Technology R\&D Project, through the Korea Health Industry Development Institute (KHIDI), funded by the Ministry of Health and Welfare (HI14C1989) and the Basic Science Research Program, through the National Research Foundation of Korea (NRF), funded by the Ministry of Education, Science and Technology (MSIT) (No. 2019M3C1B8090802, 2019M3C1B8090803 and 2020R1A2C2013216) to H.W.L., and by grants from the National Research Foundation (2020M3H4A1A03084600, and 2021R1A4A2001389) and the Korean 
Health Technology R\&D Project, Ministry of Health and Welfare (HI14C3484 and HI20C0039), and by the National Institute of Health (R01NS094388) to B.-O.C.

Institutional Review Board Statement: The study was conducted according to the guidelines of the Declaration of Helsinki and approved by the Institutional Review Board of Ewha Womans University Mokdong Hospital (approval No. ECT 14-29A-12).

Informed Consent Statement: Informed consent was obtained from all subjects involved in the study and from parents of subjects younger than 18 years of age. Written informed consent has been obtained from the patient to publish this paper.

Data Availability Statement: All of the raw and processed data for genetic and imaging analysis were stored and can be accessed in our laboratory, which is supervised by the corresponding authors, B.-O.C. and H.W.L., respectively.

Conflicts of Interest: The authors declare no conflict of interest. The funders had no role in the design of the study; in the collection, analyses, or interpretation of data; in the writing of the manuscript or in the decision to publish the results.

\section{References}

1. Shy, M.E.; Garbern, J.Y.; Kamholz, J. Hereditary motor and sensory neuropathies: A biological perspective. Lancet Neurol. 2002, 1, 110-118. [CrossRef]

2. DiVincenzo, C.; Elzinga, C.D.; Medeiros, A.C.; Karbassi, I.; Jones, J.R.; Evans, M.C.; Braastad, C.D.; Bishop, C.M.; Jaremko, M.; Wang, Z.; et al. The allelic spectrum of Charcot-Marie-Tooth disease in over 17,000 individuals with neuropathy. Mol. Genet. Genomic. Med. 2014, 2, 522-529. [CrossRef]

3. Jordanova, A.; De Jonghe, P.; Boerkoel, C.F.; Takashima, H.; De Vriendt, E.; Ceuterick, C.; Martin, J.J.; Butler, I.J.; Mancias, P.; Papasozomenos, S.; et al. Mutations in the neurofilament light chain gene (NEFL) cause early onset severe Charcot-Marie-Tooth disease. Brain 2003, 126, 590-597. [CrossRef]

4. Chung, K.W.; Suh, B.C.; Cho, S.Y.; Choi, S.K.; Kang, S.H.; Yoo, J.H.; Hwang, J.Y.; Choi, B.O. Early-onset Charcot-Marie-Tooth patients with mitofusin 2 mutations and brain involvement. J. Neurol. Neurosurg. Psychiatry 2010, 81, 1203-1206. [CrossRef]

5. Paulson, H.L.; Garbern, J.Y.; Hoban, T.F.; Krajewski, K.M.; Lewis, R.A.; Fischbeck, K.H.; Grossman, R.I.; Lenkinski, R.; Kamholz, J.A.; Shy, M.E. Transient central nervous system white matter abnormality in X-linked Charcot-Marie-Tooth disease. Ann. Neurol. 2002, 52, 429-434. [CrossRef] [PubMed]

6. Horga, A.; Laurà, M.; Jaunmuktane, Z.; Jerath, N.U.; Gonzalez, M.A.; Polke, J.M.; Poh, R.; Blake, J.C.; Liu, Y.T.; Wiethoff, S.; et al. Genetic and clinical characteristics of NEFL-related Charcot-Marie-Tooth disease. J. Neurol. Neurosurg. Psychiatry 2017, 88, 575-585. [CrossRef] [PubMed]

7. Yang, Y.; Gu, L.Q.; Burnette, W.B.; Li, J. N98S mutation in NEFL gene is dominantly inherited with a phenotype of polyneuropathy and cerebellar atrophy. J. Neurol. Sci. 2016, 365, 46-47. [CrossRef] [PubMed]

8. Ferrarin, M.; Gironi, M.; Mendozzi, L.; Nemni, R.; Mazzoleni, P.; Rabuffetti, M. Procedure for the quantitative evaluation of motor disturbances in cerebellar ataxic patients. Med. Biol. Eng. Comput. 2005, 43, 349-356. [CrossRef]

9. Berciano, J.; García, A.; Peeters, K.; Gallardo, E.; De Vriendt, E.; Pelayo-Negro, A.L.; Infante, J.; Jordanova, A. NEFL E396K mutation is associated with a novel dominant intermediate Charcot-Marie-Tooth disease phenotype. J. Neurol. 2015, 262, 1289-1300. [CrossRef]

10. Lee, M.; Park, C.H.; Chung, H.K.; Kim, H.J.; Choi, Y.; Yoo, J.H.; Yoon, Y.C.; Hong, Y.B.; Chung, K.W.; Choi, B.O.; et al. Cerebral white matter abnormalities in patients with charcot-marie-tooth disease. Ann. Neurol. 2017, 81, 147-151. [CrossRef]

11. Miltenberger-Miltenyi, G.; Janecke, A.R.; Wanschitz, J.V.; Timmerman, V.; Windpassinger, C.; Auer-Grumbach, M.; Löscher, W.N. Clinical and electrophysiological features in Charcot-Marie-Tooth disease with mutations in the NEFL gene. Arch. Neurol. 2007, 64, 966-970. [CrossRef]

12. Inada, B.S.Y.; Rezende, T.J.R.; Pereira, F.V.; Garcia LÁ, L.; da Rocha, A.J.; Neto, P.B.; Barsottini, O.G.P.; França, M.C., Jr.; Pedroso, J.L. Corticospinal tract involvement in spinocerebellar ataxia type 3: A diffusion tensor imaging study. Neuroradiology 2021, 63, 217-224. [CrossRef] [PubMed]

13. Kang, J.S.; Klein, J.C.; Baudrexel, S.; Deichmann, R.; Nolte, D.; Hilker, R. White matter damage is related to ataxia severity in SCA3. J. Neurol. 2014, 261, 291-299. [CrossRef]

14. Stezin, A.; Bhardwaj, S.; Khokhar, S.; Hegde, S.; Jain, S.; Bharath, R.D.; Saini, J.; Pal, P.K. In vivo microstructural white matter changes in early spinocerebellar ataxia 2. Acta Neurol. Scand. 2021, 143, 326-332. [CrossRef] [PubMed]

15. Wu, X.; Liao, X.; Zhan, Y.; Cheng, C.; Shen, W.; Huang, M.; Zhou, Z.; Wang, Z.; Qiu, Z.; Xing, W.; et al. Microstructural Alterations in Asymptomatic and Symptomatic Patients with Spinocerebellar Ataxia Type 3: A Tract-Based Spatial Statistics Study. Front. Neurol. 2017, 8, 714. [CrossRef] [PubMed]

16. Pontillo, G.; Dubbioso, R.; Cocozza, S.; Tozza, S.; Severi, D.; Iodice, R.; Tedeschi, E.; Elefante, A.; Brunetti, A.; Manganelli, F.; et al. Brain Plasticity in Charcot-Marie-Tooth Type 1A Patients? A Combined Structural and Diffusion MRI Study. Front. Neurol. 2020, 11, 795. [CrossRef] 
17. Paternostro-Sluga, T.; Grim-Stieger, M.; Posch, M.; Schuhfried, O.; Vacariu, G.; Mittermaier, C.; Bittner, C.; Fialka-Moser, V. Reliability and validity of the Medical Research Council (MRC) scale and a modified scale for testing muscle strength in patients with radial palsy. J. Rehabil. Med. 2008, 40, 665-671. [CrossRef]

18. Birouk, N.; LeGuern, E.; Maisonobe, T.; Rouger, H.; Gouider, R.; Tardieu, S.; Gugenheim, M.; Routon, M.C.; Léger, J.M.; Agid, Y.; et al. X-linked Charcot-Marie-Tooth disease with connexin 32 mutations: Clinical and electrophysiologic study. Neurology 1998, 50, 1074-1082. [CrossRef]

19. Shy, M.E.; Blake, J.; Krajewski, K.; Fuerst, D.R.; Laura, M.; Hahn, A.F.; Li, J.; Lewis, R.A.; Reilly, M. Reliability and validity of the CMT neuropathy score as a measure of disability. Neurology 2005, 64, 1209-1214. [CrossRef]

20. Schmitz-Hübsch, T.; Du Montcel, S.T.; Baliko, L.; Berciano, J.; Boesch, S.; Depondt, C.; Giunti, P.; Globas, C.; Infante, J.; Kang, J.S.; et al. Scale for the assessment and rating of ataxia: Development of a new clinical scale. Neurology 2006, 66, 1717-1720. [CrossRef]

21. Forbes, J.L.; Kim, R.E.; Paulsen, J.S.; Johnson, H.J. An Open-Source Label Atlas Correction Tool and Preliminary Results on Huntingtons Disease Whole-Brain MRI Atlases. Front. Neuroinform. 2016, 10, 29. [CrossRef]

22. Kim, E.Y.; Magnotta, V.A.; Liu, D.; Johnson, H.J. Stable Atlas-based Mapped Prior (STAMP) machine-learning segmentation for multicenter large-scale MRI data. Magn. Reson. Imaging 2014, 32, 832-844. [CrossRef] [PubMed]

23. Kim, R.E.; Lourens, S.; Long, J.D.; Paulsen, J.S.; Johnson, H.J. Preliminary analysis using multi-atlas labeling algorithms for tracing longitudinal change. Front. Neurosci. 2015, 9, 242. [CrossRef]

24. Wang, H.; Yushkevich, P.A. Multi-atlas segmentation with joint label fusion and corrective learning-an open source implementation. Front. Neuroinform. 2013, 7, 27. [CrossRef] [PubMed]

25. Kim, S.E.; Lee, J.H.; Chung, H.K.; Lim, S.M.; Lee, H.W. Alterations in white matter microstructures and cognitive dysfunctions in benign childhood epilepsy with centrotemporal spikes. Eur. J. Neurol. 2014, 21, 708-717. [CrossRef]

26. Smith, S.M.; Jenkinson, M.; Johansen-Berg, H.; Rueckert, D.; Nichols, T.E.; Mackay, C.E.; Watkins, K.E.; Ciccarelli, O.; Cader, M.Z.; Matthews, P.M.; et al. Tract-based spatial statistics: Voxelwise analysis of multi-subject diffusion data. Neuroimage 2006, 31, 1487-1505. [CrossRef]

27. Abrams, C.K.; Freidin, M. GJB1-associated X-linked Charcot-Marie-Tooth disease, a disorder affecting the central and peripheral nervous systems. Cell Tissue Res. 2015, 360, 659-673. [CrossRef]

28. Wang, W.; Zhang, F.; Li, L.; Tang, F.; Siedlak, S.L.; Fujioka, H.; Liu, Y.; Su, B.; Pi, Y.; Wang, X. MFN2 couples glutamate excitotoxicity and mitochondrial dysfunction in motor neurons. J. Biol. Chem. 2015, 290, 168-182. [CrossRef] 